\title{
Comprehensive Analysis of Epigenetic Alternations of MicroRNA and DNA Methylation Contribute to Gene Expression and Prognosis in Lung Squamous Cell Carcinoma
}

\author{
Xiaoming Li \\ Beijing Yanhua Hospital \\ Yingli Liang \\ Beijing Yanhua Hospital \\ Huixin Wang ( $\sim$ I19910292800@163.com) \\ Beijing Fangshan Traditional Chinese Medicine Hospital
}

\section{Research Article}

Keywords: Lung cancer, miRNAs, differentially expressed genes, regulation, hypermethylation, prognosis

Posted Date: February 18th, 2022

DOI: https://doi.org/10.21203/rs.3.rs-1363706/v1

License: (c) (1) This work is licensed under a Creative Commons Attribution 4.0 International License.

Read Full License 


\section{Abstract \\ Background}

Early detection of lung squamous cell carcinoma (LUSC) is significantly effective in clinical management. This study aimed to identify potential LUSC biomarkers based on the epigenetic alterations of miRNAs and DNA methylation.

\section{Methods}

Analysis of expression profiles in LUSC clinical samples downloaded from The Cancer Genome Atlas (TCGA-LUSC) datasets was performed to identify differentially expressed genes (DEGs) using $R$ packages. The biological functions were annotated by Gene Ontology (GO) and Kyoto Encyclopedia of Genes and Genomes (KEGG) enrichment analysis. The protein-protein interaction (PPI) network of the DEGs was established through STRING (Search Tool for the Retrieval of Interacting Genes database) website, visualized by Cytoscape and further analyzed by Molecular Complex Detection (MCODE). Kaplan-meier method and univariate Cox proportional risk regression analysis were used to screen differential genes related to survival in LUSC patients. Multivariate Cox proportional risk regression analysis was used to construct a prognosis prediction model of LUSC patients with differentially expressed genes. Receiver operating characteristic (ROC) curve analysis confirmed the biomarker's specific and sensitivity.

\section{Results}

We systematically identified 96 DEGs modified by both DNA methylation and miRNA through TCGA dataset between LUSC tissues and normal tissues. KEGG pathway and GO enrichment analysis gave us an insightful view of the functions of DEMs and DMGs. Subsequently, the four-gene prognosis model were obtained, including SMAD7, MEF2C, TCF21, and TRIB1, together with clinical factors, smoking history and EvsA. Survival curve analysis indicates that the model has good predictive value for the prognosis of patients with LUSC. We also found their differential expression, especially MEF2C and TCF21, together with immune infiltration levels in B cells, CD8 + T cells, CD4 + T cells, macrophages, neutrophils, and dendritic cells, correlated with LUSC prognosis.

\section{Conclusions}

Four DEGs modified by both DNA methylation and miRNA were associated with the overall survival of LUSC patients and could be potential biomarkers to predict prognosis. MEF2C and TCF21 likely play important roles in immune cell infiltration and as prognosis biomarkers in LUSC patients with immune 
cell infiltration. In future research, we will explore the prognostic genes and their potential function based on the present study.

\section{Background}

Lung cancer is a leading cause of cancer-associated mortality worldwide, and the incidence and mortality rates of lung cancer have significantly increased. Lung squamous cell carcinoma (LUSC) is the common histological type of lung cancer [1]. Despite advances in multiple treatments including surgical resection, chemotherapy, radiotherapy, and molecular targeted therapy, the prognosis of LUSC remains dismal $[2,3]$. A limited number of biomarkers are available for diagnosing LUSC $[4,5]$. Therefore, it is critical to focus on biomarker combinations that encompass a variety of different pathways to improve the prognoses of the patients with LUSC and develop novel targeted strategies to treat LUSC.

Epigenetic regulation, such as DNA methylation and histone modifications, refers to changing the chromatin structure, regulating gene expression, and controlling cellular development and differentiation; however, it does not depend on the variations in the DNA sequence [6]. The epigenetic modifiers are categorized as "writers", "readers", and "erasers", which add chemical tags on DNA and histones, recognize and interpret the specific modifications, and remove these chemical tags [7]. Epigenetic alterations have been regarded as crucial regulatory factors that are involved in numerous disease conditions, especially participating in tumorigenesis and cancer cell phenotypes [8]. Global alterations at $\mathrm{CpG}$ dinucleotides in DNA methylation through adding methyl groups mediated by DNA methyltransferases have been established in $\mathrm{HCC}$ and are considered as prognostic biomarkers [7, 9]. DNA methylation is the phenomenon that methyl groups bound to cytosine where the nucleotide is directly followed by a guanine (CpG islands) $[10,11]$. CpG island methylation usually affects multiple genes including tumor suppressor, thus participating in the development and progression of cancer.

MicroRNAs (miRNAs) are short, approximately 22 nucleotides in length that have no ability of coding proteins and have been suggested to represent transcriptional noise, which widely exits in high eukaryotes $[12,13]$. However, more and more evidence indicates that miRNAs play an important role in regulating genes associated with malignant biological behavior in cancer cells [14, 15]. After biogenesis, miRNAs combine the untranslated region (UTR) of the messenger RNAs (mRNAs) of their cognate target genes [16]. MiRNAs as oncogenes (onco-miRNAs) or tumor suppressors (ts-miRNAs) by inhibiting the expression of target genes via cleaving the mRNA molecules or inhibiting their translation, suggests their potential as diagnostic markers of malignancy $[17,18]$. Until now, however, no comprehensive regulatory network has been profiled about how epigenetic alternations of microRNAs and DNA methylation modulate LUSC.

In the present study, we conducted a multi-step analysis using various $\mathrm{R}$ language packages on clinical samples downloaded from The Cancer Genome Atlas (TCGA) database to identify differentially expressed genes (DEGs), miRNAs (DEMs), and differentially methylated genes (DMGs) [19]. The DEGs effected by both DNA methylation and miRNA were also obtained. Then, the DEGs significantly 
associated with patients' overall survival were identified as patient prognosis based on the Cox regression method.

\section{Materials And Methods Data collection}

Genome-wide DNA methylation, miRNA expression, and clinical information of the patients were retrieved from TCGA-LUSC data portal (https://portal.gdc.cancer.gov/), including 382 LUSC tissues and 31 matched normal tissues. The platforms for DNA methylation were Human Methylation 450 with 485,577 probes. According to the clinical pathological stage (I IV) of LUSC of American Joint Committee (AJCC), the patient sample data was divided into early-stage cases and advanced-stage cases, which respectively contained 178 and 186. The detailed clinical characteristics of patients are listed in Table S1.

\section{Data processing}

The DEMs and DEGs between LUSC and normal lung tissues of the TCGA-LUSC dataset, were calculated using the limma package in R [20]. Adjusted $P$-value $<0.05$ and $\mid \log _{2}$ fold change (FC) $\mid>1.0$ and 1.5 were set as cut-off criteria, respectively. Volcano plot and heatmap of the identified DEMs and DEGs were visualized using ggplot2 and pheatmap packages of $R$, respectively [21]. The methylation level of $\mathrm{CpGs}$ was represented as $\beta$ values $(\beta=$ Intensity of the methylated allele $(M) /[$ Intensity of the unmethylated allele $(U)+$ Intensity of the methylated allele $(M)+100$ ], ranging from 0 to 1$)$. Then, differentially methylated CpGs were identified between the LUSC and normal tissue using the limma package in R. $P$ values were adjusted using the false discovery rate (FDR) method. The methylated $\mathrm{CpGs}$ located genes with an FDR $<0.05$ and an absolute value of the $\beta$ difference $>0.2$ were considered as DMGs.

\section{GO and KEGG pathway enrichment analysis}

DEM-targets were obtained from the reliable online databases, including miRDB, TargetScan, miRTarBase, etc., using the "multiMiR" package in R (http://multimir.org/) [22]. To better understand the biological functions of the DEMs-targets and DMGs, Gene Ontology (GO) covering biological processes (BP), molecular functions (MF), and cellular components (CC), and Kyoto Encyclopedia of Genes and Genomes (KEGG) pathway enrichment analyses were conducted using the clusterProfile package in R, respectively [23-25]. An adjusted $P$-value less than 0.05 and a count more than 2 were considered as cut-off point criteria, and the results were visualized with the ggplot2 package in $\mathrm{R}$.

\section{Protein-protein interaction analysis}

The STRING v.11.5 online tool (http://string-db.org/) [26] was used to analyze the protein-protein interactions (PPI) among the DEGs co-regulated by miRNA and DNA methylation. Then, integration of networks was visualized using Cytoscape software. The Molecular Complex Detection (MCODE) was applied to screen modules of PPI network with degree cutoff $=2$, node score cutoff $=0.2, k$-core $=2$, and max. depth $=100$. Subsequently, the enrichment analysis of DEGs in top three clusters was carried out. 


\section{Identification of DEGs co-regulated by miRNA and DNA methylation}

Down-regulated DEMs targeting up-regulated DEGs and up-regulated DEMs targeting down-regulated DEGs were obtained by overlapping DEGs and potential targets of DEMs, respectively. Meanwhile, we summarized hypomethylation-high-expression genes through overlapping hypomethylated DMGs and upregulated DEGs and got hypermethylation-low-expression genes via overlapping hypermethylated DMGs and down-regulated DEGs. According to visualization performed by the VennDiagram package of R [27], and the overlapped genes were identified as the DEGs co-regulated by miRNA and DNA methylation. Furthermore, the miRNA-mRNA and DNA methylation-mRNA interactions were integrated to establish a dysregulated network and visualize using Cytoscape software [28].

\section{Survival analysis}

To identify the prognosis of the DEGs co-regulated by miRNA and DNA methylation, their expression profiles were normalized by log2 transformation and analyzed with univariate Cox regression ( $P$-value < 0.05). The hazard ratios (HRs) with $95 \%$ confidence intervals $(\mathrm{Cl})$ were also computed. The median value of expression levels was applied as a cut-off to divide patients into high- and low-level groups. The genes that were significantly associated with overall survival (OS) of patients with LUSC were identified.

Survival curves of the genes were evaluated and plotted using the Kaplan-Meier method and the Log-rank test by the survival package in $\mathrm{R}$ (https://CRAN.R-project.org/package=survival). The Log-rank test was used to evaluate statistical significance with a cut-off criterion of $P<0.05$.

\section{Cox risk regression model construction}

Through the R language software Survival and Survminer packages, the DEGs co-regulated by miRNA and DNA methylation obtained in the previous step were analyzed by single factor Cox proportional risk regression, and the DEGs with statistical significance $(P<0.01)$ were screened out. Furthermore, the multifactor Cox proportional risk regression was used to screen out the genes related to the prognosis of LUSC patients $(P<0.05)$. The prediction model of patients' OS was established, and the prognostic index $(\mathrm{PI})$ was calculated. According to the median score, patients were divided into high-risk group and low-risk group. Log-rank test was performed by Kaplan-Meier (K-M) method for survival analysis. The validity and sensitivity of polygenic prognosis model were evaluated by calculating the area under the curve (AUC) of receiver operating characteristic (ROC) curve of subjects.

\section{Correlation of DEGs in prognosis model in TILs of LUSC}

The correlation between expression of the DEGs co-regulated by miRNA and DNA methylation and the major immune cells, including B cell, CD4 + T cell, CD $8+T$ cell, neutrophil, macrophage, monocyte cell, etc., was explored via TIMER database (https://cistrome.shinyapps.io/timer/) with $P$-value $<0.05$ as significance.

\section{Statistical analysis}


All analyses were conducted using RStudio software (Version 1.3.959). The Student's $t$ test was conducted to compare the differences. And a two-sided $P<0.05$ was regarded as significant.

\section{Results}

\section{Identification of DEMs and DMGs between LUSC and normal tissues}

A total of 146 DEMs between LUSC and normal samples were obtained after analyzing miRNA expression profiles from TCGA-LUSC with R language using adjusted $P$-value $<0.05$ and $\| \log _{2} \mathrm{FC} \mid>1.0$ as screening criteria. Among them, 52 miRNAs were significantly downregulated in LUSC tissues compared with normal tissues, and 94 miRNAs were significantly upregulated. The volcano plot and heatmap illustrated the significant differences and distribution of the fold change in DEMs (Fig. 1A, B). A total of 26511 DNA methylation loci were differentially methylated between LUSC tissues and noncancerous lung tissues. Among them, 10755 and 15756 were hypermethylated and hypomethylated in tumor samples, respectively, compared with normal lung tissues $(P<0.05$, Fig. 1C, D).

\section{Genes associated with altered targeting miRNAs}

GO analysis, which was performed to determine the biological functions of the targets of up-regulated DEMs (Table S2), showed that these genes were significantly mainly enriched in response to oxygen levels (G0:0070482, $P=6.21 \mathrm{E}-48)$, gland development (G0:0048732, $P=6.21 \mathrm{E}-48$ ), response to decreased oxygen levels (G0:0036293, $P=2.40 \mathrm{E}-42)$, transcription factor complex (G0:0005667, $P=$ 2.16E-26), nuclear chromatin (G0:0000790, $P=1.59 \mathrm{E}-23)$, membrane microdomain (G0:0098857, $P=$ 1.88E-16), ubiquitin-like protein ligase binding (G0:0044389, $P=7.05 \mathrm{E}-18)$, enhancer binding (G0:0035326, $P=5.34 \mathrm{E}-15)$, and growth factor binding (G0:0019838, $P=2.12 \mathrm{E}-14$ ) (Fig. 2A, Table S3). Meanwhile, the biological functions of the targets of down-regulated DEMs were significantly mainly enriched in response to epithelial cell proliferation (G0:0050673, $P=2.47 \mathrm{E}-42)$, gland development (GO:0048732, $P=6.49 \mathrm{E}-41)$, response to oxygen levels (G0:0070482, $P=9.30 \mathrm{E}-41$ ), focal adhesion (GO:0005925, $P=4.68 \mathrm{E}-20)$, cell-substrate adherens junction (G0:0005924, $P=4.78 \mathrm{E}-20)$, cell leading edge (GO:0031252, $P=1.00 \mathrm{E}-14)$, cell adhesion molecule binding (GO:0050839, $P=2.00 \mathrm{E}-18)$, growth factor binding (G0:0019838, $P=3.35 \mathrm{E}-16)$, and ubiquitin-like protein ligase binding $(\mathrm{GO}: 0031625, P=$ 3.14E-14) (Fig. 2A, Table S3).

KEGG pathway analysis revealed that the up-regulated DEMs-targets were mainly involved in AGE-RAGE signaling pathway in diabetic complications (hsa04933, $P=1.78 \mathrm{E}-20$ ), PI3K-Akt signaling pathway (hsa04151, $P=1.20 \mathrm{E}-19)$, EGFR tyrosine kinase inhibitor resistance (hsa01521, $P=8.47 \mathrm{E}-19$ ), Human papillomavirus infection (hsa05165, $P=1.19 \mathrm{E}-15$ ), and MAPK signaling pathway (hsa04010, $P=1.80 \mathrm{E}-$ 16) $(P<0.01$, Fig. 2B, Table S3). The down-regulated DEMs-targets were mainly involved in Human papillomavirus infection (hsa05165, $P=7.88 \mathrm{E}-32$ ), MicroRNAs in cancer (hsa05165, $P=3.39 \mathrm{E}-38$ ), FoxO 
signaling pathway (hsa04068, $P=1.2 \mathrm{E}-30$ ), Cellular senescence (hsa04218, $P=7.86 \mathrm{E}-27$ ), and Focal adhesion (hsa04510, $P=2.64 \mathrm{E}-27)(P<0.01$, Fig. 2B, Table S4).

\section{Genes associated with altered DNA methylation}

GO analysis, which was performed to determine the biological functions of the hypermethylation associated genes (Table S5), showed that these genes were significantly mainly enriched in regionalization (G0:0003002, $P=1.59 \mathrm{E}-51)$, pattern specification process (G0:0007389, $P=1.59 \mathrm{E}-51)$, embryonic organ development (G0:0048568, $P=6.32 \mathrm{E}-46)$, synaptic membrane (G0:0097060, $P=8.69 \mathrm{E}-$ 15), transcription factor complex (G0:0005667, $P=1.39 \mathrm{E}-14)$, postsynaptic membrane (G0:0045211, $P=$ 9.00E-14), DNA-binding transcription activator activity, RNA polymerase II-specific (G0:0001228, $P=$ 8.24E-39), gated channel activity (G0:0022836, $P=4.99 \mathrm{E}-08)$, and ion gated channel activity (G0:0022839, $P=4.99 \mathrm{E}-08$ ). Meanwhile, the biological functions of the hypomethylation associated genes were significantly mainly enriched in sensory perception of smell (G0:0007608, $P=2.55 \mathrm{E}-27)$, modulation of chemical synaptic transmission (G0:0050804, $P=1.14 \mathrm{E}-25)$, regulation of trans-synaptic signaling (GO:0099177, $P=1.29 \mathrm{E}-25)$, transmembrane transporter complex (G0:1902495, $P=2.20 \mathrm{E}-25)$, transporter complex (G0:1990351, $P=1.18 \mathrm{E}-24)$, ion channel complex (G0:0034702, $P=1.18 \mathrm{E}-24)$, ion channel activity (G0:0005216, $P=2.86 \mathrm{E}-26)$, olfactory receptor activity (G0:0004984, $P=5.15 \mathrm{E}-26)$, and passive transmembrane transporter activity (G0:0022803, $P=1.18 \mathrm{E}-25)$ (Fig. 3A, Table S6).

KEGG pathway analysis revealed that the hypermethylation associated genes were mainly involved in CAMP signaling pathway (hsa04024, $P=1.08 \mathrm{E}-10)$, neuroactive ligand-receptor interaction (hsa04080, $P$ $=6.59 \mathrm{E}-10$ ), signaling pathways regulating pluripotency of stem cells (hsa04550, $P=3.37 \mathrm{E}-07$ ), adrenergic signaling in cardiomyocytes (hsa04261, $P=5.04 \mathrm{E}-07$ ), and parathyroid hormone synthesis, secretion and action (hsa04928, $P=8$.29E-07) (Fig. 3B, 4A, Table S7). The hypomethylation associated genes were mainly involved in olfactory transduction (hsa04740, $P=6.76 \mathrm{E}-28$ ), arrhythmogenic right ventricular cardiomyopathy (hsa05412, $P=4$.87E-08), calcium signaling pathway (hsa04020, $P=5.14 \mathrm{E}-$ 07), neuroactive ligand-receptor interaction, and Insulin secretion (hsa04080, $P=6.21 \mathrm{E}-07$ ) (Fig. 3B, 4B, Table S7).

\section{DEGs associated with both aberrant miRNA and DNA methylation}

Meanwhile, DEGs were also obtained between LUSC and normal tissues, including 2894 down-regulated DEGs and 1340 up-regulated DEGs using adjusted $P$-value $<0.05$ and $\left|\log _{2} \mathrm{FC}\right|>1.5$ as screening criteria (Fig. S1). Interestingly, some DEGs were affected by both aberrant alternations of miRNA and DNA methylation, which might demonstrate valuable information underlying LUSC. Twenty-six genes, including SLC2A1, KIFC1, CDC25A, MCM2, HMGA1, COL7A1, FAM83F, MMP11, ARTN, GAPDH, etc. were upregulated under the modulation of both decreased miRNA and hypomethylation (Fig. 5A). Seventy genes of TCF21, SOX17, TMEM88, PRKCE, MAMDC2, LEPR, GPD1L, LRRK2, DOCK4, PTPRM, NR0B2, KDR, HECW2, DUSP1, GATA6, etc. were down-regulated under modulation of both increased miRNA and hypermethylation (Fig. 5B). As shown in Fig. S2, expression of 25 DEGs were affected by more than five 
DNA methylation loci each, and 15 DEGs were regulated by more than two miRNAs. In addition, the PPI network of the obtained DEGs is consisted of 96 nodes and 193 edges. Three significant modules were obtained from PPI network of DEGs using MCODE (Fig. S2). Cluster 1 contained eleven DEGs, STAT5A, SOST, MMP13, BMP2, SLC2A1, LEPR, PPARGC1A, GAPDH, TIMP2, TERT and SPP1. Cluster 2 contained seven DEGs, SOX17, FOXA2, MEF2C, GATA6, JAG1, TGFB2, and ZEB2. Cluster 3 contained five DEGs, ETS1, SOX7, DAB2, ESR1, and KDR.

\section{Establishment of Cox prognostic model}

Univariate Cox regression analysis was used to analyze 96 DEGs affected by both aberrant alternations of miRNA and DNA methylation in TCGA-LUSC dataset. A total 22 genes together with clinical factors, including TNM stage, tumor size, smoking history of patients, and Early vs Advanced stages (EvsA), were screened out according to $P<0.01$, which may affect the overall survival time of patients. Furthermore, the four genes together with smoking history, and EvsA were obtained by multivariate Cox proportional hazard regression analysis by two-way stepwise regression, including SMAD7, MEF2C, TCF21, and TRIB1, and the prognostic prediction model was established (Fig. 6). The prognostic score formula was as follows: $\mathrm{PI}=-0.293 \times$ smoking history $-0.570 \times$ EvsA $-0.257 \times \mathrm{MEF} 2 \mathrm{C}$ expression quantity $+0.200 \times$ SMAD7 expression quantity $+0.214 \times$ TCF21 expression quantity $+0.268 \times$ TRIB 1 expression quantity. According to the median RS value (1.037) calculated by the prognostic score formula, 364 patients were divided into low-risk group and high-risk group. As shown in Fig. 6A, the survival rate in the low-risk group was significantly higher than that in the high-risk group (Logrank $P<0.0001$ ). As shown in Fig. 6B, the AUC value of the ROC curve was 0.643 indicating that the polygene prognostic model had good sensitivity and specificity. The 1-year, 3-year and 5-year survival of the patients were analyzed, and the time-dependent ROC curve AUC values of the polygene prognostic model were $0.647,0.698$ and 0.724 , respectively, indicating that the prognostic model had a good ability to predict the prognosis (Fig. $6 \mathrm{C}$ ). The higher the prognosis score, the more the number of deaths, indicating that the higher the risk score, the worse the prognosis. Figure 6D showed the expression of genes in high and low risk groups in the polygenic prognostic model.

Among them, all the four genes had significantly lower mRNA expression and higher DNA methylation level in LUSC tissues compared with in normal tissues $(P<0.001$, Fig. S3A, B). We also found except TRIB1, the other three genes were significantly downregulated in advanced stage of LUSC patients compared with early stage of LUSC patients $(P<0.05$, Fig. S3C). Moreover, extremely differences of MEF2C and SMAD7 expression between current smokers and reformed smokers in LUSC patients $(P<$ 0.01 , Fig. S3D).

The correlation between the expression of these four DEGs and the LUSC patients' prognostic outcomes was evaluated by Kaplan-Meier analysis in the TCGA dataset. Patients were divided into high and low expression groups based on the median expression value. As shown in Fig. 7, lower expression of SMAD7, TCF21, and TRIB1 of LUSC patients (early stage, advanced stage, or reformed smokers) had significantly better OS, respectively $(P<0.01)$. In addition, lower expression of SMAD7 and TCF21 of current smoking LUSC patients had significantly better OS, respectively $(P<0.01)$. While as lower 
expression of MEF2C of reformed smoking LUSC patients had significantly better OS $(P<0.05)$. These results indicated that SMAD7 and TCF21 could be potential independent biomarkers for LUSC diagnosis.

\section{The sites of DEGs co-regulated by miRNA and DNA methylation in prognosis model}

The specific regulatory miRNA and binding site, as well as the DNA methylation site and its relation to CpG island, and the representative epigenetic regulatory pattern of both miRNA and DNA methylation on gene expression was shown in Fig. 8. DNA hypermethylation at cg04694437 on 5'UTR of MEF2C, as well as higher expressed miRNAs, hsa-mir-21 and hsa-mir-135b, were significantly and negatively correlated with mRNA expression of MEF2C. DNA hypermethylation at cg07661480 on 3'UTR and CpG loci including cg13398027, cg24375218, cg11909137, and cg20800216 on the sequence body of SMAD7, as well as higher expressed miRNA (e.g. hsa-mir-21, hsa-mir-93, hsa-mir-424, etc.) were significantly and negatively correlated with mRNA expression of SMAD7. DNA hypermethylation at various CpG loci on the TSS200, 1st Exon, sequence body, and 3'UTR of TCF21 (e.g. cg12359158, cg04467618, cg15794859, etc.) as well as higher expressed hsa-mir-21 were significantly and negatively correlated with mRNA expression of TCF21. DNA hypermethylation at cg02160684 on the sequence body of TRIB1, as well as higher expressed hsa-mir-224 were significantly and negatively correlated with mRNA expression of TRIB1 (Table S8, Fig. S4).

\section{DEGs co-regulated by miRNA and DNA methylation were related to immune infiltration of LUSC}

As immune infiltration of tumor potentially affects patient's survival. Herein, we analyzed the potential correlation between the expression of DEGs co-regulated by miRNA and DNA methylation, tumor purity, and immune infiltration in the online tool TIMER. The correlation of their expression with LUSC tumor purity and immune infiltration was revealed (Fig. S5). The expression of MEF2C and TCF21 had a positive correlation with infiltrating levels of $\mathrm{B}$ cells $(r=0.51, P=1.47 \mathrm{e}-32 ; r=0.323, P=6.35 \mathrm{e}-13)$, CD8 $+\mathrm{T}$ cells $(r=0.488, P=8.79 \mathrm{e}-30 ; r=0.306, P=9.16 \mathrm{e}-12)$, CD $4+\mathrm{T}$ cells $(r=0.501, P=1.46 \mathrm{e}-31 ; r=$ 0.486, $P=1.39 \mathrm{e}-29)$, macrophages $(r=0.597, P=2.62 \mathrm{e}-47 ; r=0.588, P=9.45 \mathrm{e}-46)$, neutrophils $(r=$ $0.549, P=7.65 \mathrm{e}-39 ; r=0.387, P=1.74 \mathrm{e}-18)$, and dendritic cells $(r=0.7, P=6.60 \mathrm{e}-71 ; r=0.505, P=$ $4.12 \mathrm{e}-32)$ in LUSC, respectively. The expression of SMAD7 had a positive correlation with infiltrating levels of CD4 $+\mathrm{T}$ cells $(r=0.538, P=4.99 \mathrm{e}-37)$, macrophages $(r=0.411, P=7.77 \mathrm{e}-21)$, neutrophils $(r=$ $0.46, P=2.44 \mathrm{e}-26)$, and dendritic cells $(r=0.533, P=3.22 \mathrm{e}-36)$ in LUSC, respectively, but had low positive relationship with B cells $(r=0.197, P=1.58 \mathrm{e}-05)$ and CD8 + T cells $(r=0.234, P=2.36 \mathrm{e}-07)$. The expression of TRIB1 had a positive correlation with infiltrating levels of CD4 $+\mathrm{T}$ cells $(r=0.333, P=$ $9.27 \mathrm{e}-14)$, and neutrophils ( $r=0.412, P=6.16 \mathrm{e}-21)$ in LUSC, respectively, had low positive relationship with CD $8+\mathrm{T}$ cells $(r=0.197, P=1.46 \mathrm{e}-05)$, macrophages $(r=0.256, P=1.49 \mathrm{e}-08)$, and dendritic cells $(r$ $=0.268, P=3.08 \mathrm{e}-09)$, however, had no relationship with $\mathrm{B}$ cells $(r=0.003, P=9.41 \mathrm{e}-01)$.

\section{Discussion}


The epigenetic modifiers have been highlighted for critical functions in cancer initiation and progression. To identify the epigenetic molecular mechanism participant in LUSC tumorigenesis and to investigate the potential biomarkers for better detection and therapy, we analyzed the gene expression profiles and global DNA methylation of the TCGA-LUSC dataset.

We identified plenty of DEMs and DMGs between LUSC and normal tissues. To assess the biological effects of the DEMs-targets and DNA methylation associated genes, GO and KEGG functional enrichment analysis was performed. GO enrichment analysis revealed that the genes were mainly enriched in DNA conformation change, covalent chromatin modification, and chromosomal region. Meanwhile, KEGG pathway analysis suggested that the signal pathways including cell cycle, homologous recombination, oocyte meiosis were significantly involved. Notably, these pathways have been shown to be related to the cancer's occurrence and development [29-31]. Our results suggested that the prognosis of LUSC may be improved by testing the identified DEMs and DMGs, and the LUSC development is a complex process involving various genes and proteins.

Importantly, we identified 96 DEGs were co-affected by aberrant alternations of miRNA and DNA methylation. To investigate the prognostic significance of the DEGs, we constructed the Cox regression model, and performed Kaplan-Meier and ROC analysis. Univariate and multivariate Cox proportional hazard regression analysis of differentially expressed genes was carried out by two-way stepwise regression, and four genes were obtained, including SMAD7, MEF2C, TCF21, and TRIB1, together with clinical factors, smoking history and EvsA, and a multi-gene prognostic risk score model was established. Survival curve analysis indicates that the model has good predictive value for the prognosis of patients with LUSC. At the same time, time-dependent ROC curve analysis shows that the model has good predictive efficiency.

SMAD7, is located on18q21.1 and a major negative regulator of the TGF- $\beta$ signaling pathway [32]. The regulatory factors involved in the transcriptional regulation of Smad7, such as TGF- $\beta$, epidermal growth factor (EGF) and inflammatory cytokines, such as TNF-a, IL-1 $\beta$ and IFN- $\gamma$, as well as posttranscriptional regulation including ubiquitination, acetylation, methylation, long noncoding RNAs (IncRNAs), and miRNAs (e.g. miRNA-21, miRNA-590, miRNA-497, etc.) [33-35]. Moreover, Smad7 participates in the differentiation of $T$ cells and promotes activation of immune cells by negatively regulating the TGF- $\beta$ pathway and may thereby mediate the progression of immune diseases [36]. Mads/Mef2 (Mef2a/b/c/d) family, the lineage-specific transcription factors (TFs) regulate differentiation of muscle cells, neurons and hematopoietic cells, are often deregulated in cancers. MEF2C was reported as a new molecular target in ferroptosis-inducing therapies for meningioma according to MEF2C silencing enhanced Erastininduced ferroptotic meningioma cell death and lipid peroxidation levels[37]. Mef2c, Mef2d, together with Hdac9 controlled the immunosuppressive functions of CD4 + Foxp3 + T-regulatory cells [38]. MEF2C was also explored regulated by miRNAs, including miR-802, miR-194, and miR-190b $[39,40]$. Transcription factor 21 (TCF21), a member of the basic helix-loop-helix (bHLH) transcription factors, was methylated and downregulated and inhibited gastric cancer growth and chemoresistance possibly through the AKT signaling pathway in and served as an independent prognostic factor [41]. Promoter methylation of 
TCF21 was frequently observed and might repress autophagy in the early stage of non-small cell lung cancer (NSCLC) [42]. The tribbles homologue 1 gene (TRIB1) has recently shown to be a potential diagnostic and therapeutic target and promote colorectal cancer (CRC) cell motility by activation MMP-2 via the FAK/Src and ERK pathways [43]. Aberrant transcription of TRIB1 controlled by CMYC is causal to prostate cancer pathogenesis based on the mouse modeling and functional analysis [44]. The expression of TRIB1 was significantly correlated with the infiltration of CD163 + macrophages in prostate cancer [45].

In our study, we also found their differential expression, especially MEF2C and TCF21, together with immune infiltration levels in B cells, CD8 + T cells, CD4 + T cells, macrophages, neutrophils, and dendritic cells, correlated with LUSC prognosis. Therefore, MEF2C and TCF21 likely play important roles in immune cell infiltration and as prognosis biomarkers in LUSC patients with immune cell infiltration. The above studies support the effect of the multi-gene prognostic risk model on LUSC. In this study, the multi-gene prognostic risk model constructed by bioinformatics has not been reported, and its function is worthy of further study. The model has a good effect in predicting the prognosis of LUSC and can be used as a combination of potential markers to predict the prognosis of LUSC and provide data support for finding corresponding targeted therapeutic drugs. As this study is based on the prediction model of LUSC data set in TCGA database, we need to collect large samples of clinical data to verify the effectiveness and reliability of the model.

\section{Conclusion}

We systematically identified 96 DEGs modified by both DNA methylation and miRNA through TCGA dataset between LUSC tissues and normal tissues. KEGG pathway and GO enrichment analysis gave us an insightful view of the functions of DEMs and DMGs. Subsequently, we got four-gene prognosis model were obtained, including SMAD7, MEF2C, TCF21, and TRIB1, together with clinical factors, smoking history and EvsA. Survival curve analysis indicates that the model has good predictive value for the prognosis of patients with LUSC. In future research, we will explore the prognostic genes and their potential function based on the present study.

\section{Declarations}

\section{Availability of data and materials}

The datasets used during the present study are available from the corresponding author upon reasonable request.

\section{Acknowledgements}

We gratefully acknowledge the TCGA project organizers as well as all study participants for making the data and results available.

\section{Conflict of interest}


The authors declare that they have no competing interests.

\section{Authors' contributions}

$\mathrm{HW}, \mathrm{XL}$ and $\mathrm{LL}$ participated in the design of this study, $\mathrm{XL}$ and $\mathrm{LL}$ performed the statistical analysis, $\mathrm{XL}$ drafted the manuscript, HW, and LL revised the manuscript. All authors read and approved the final manuscript.

\section{Ethics approval and consent to participate}

Not applicable.

\section{Consent for publication}

Not applicable.

\section{Funding}

Not applicable.

\section{References}

1. Conti L, Gatt S. Squamous-Cell Carcinoma of the Lung. N Engl J Med. 2018;379:e17.

2. Cassim S, Chepulis L, Keenan R, Kidd J, Firth M, Lawrenson R. Patient and carer perceived barriers to early presentation and diagnosis of lung cancer: a systematic review. BMC Cancer [Internet]. 2019 [cited 2021 Dec 23];19:25. Available from: https://www.ncbi.nlm.nih.gov/pmc/articles/PMC6323678/

3. Jensen AR, Mainz J, Overgaard J. Impact of delay on diagnosis and treatment of primary lung cancer. Acta Oncol. 2002;41:147-52.

4. Xu F, Lin H, He P, He L, Chen J, Lin L, et al. A TP53-associated gene signature for prediction of prognosis and therapeutic responses in lung squamous cell carcinoma. Oncoimmunology [Internet]. 2020 [cited 2021 Dec 23];9:1731943. Available from: https://www.ncbi.nlm.nih.gov/pmc/articles/PMC7051188/

5. Gao M, Kong W, Huang Z, Xie Z. Identification of Key Genes Related to Lung Squamous Cell Carcinoma Using Bioinformatics Analysis. Int J Mol Sci. 2020;21:E2994.

6. Nowacka-Zawisza M, Wiśnik E. DNA methylation and histone modifications as epigenetic regulation in prostate cancer (Review). Oncol Rep. 2017;38:2587-96.

7. Hattori N, Ushijima T. Compendium of aberrant DNA methylation and histone modifications in cancer. Biochem Biophys Res Commun. 2014;455:3-9.

8. Okugawa Y, Grady WM, Goel A. Epigenetic Alterations in Colorectal Cancer: Emerging Biomarkers. Gastroenterology. 2015;149:1204-1225.e12. 
9. Hao X, Luo H, Krawczyk M, Wei W, Wang W, Wang J, et al. DNA methylation markers for diagnosis and prognosis of common cancers. Proc Natl Acad Sci U S A. 2017;114:7414-9.

10. Cheng J, Wei D, Ji Y, Chen L, Yang L, Li G, et al. Integrative analysis of DNA methylation and gene expression reveals hepatocellular carcinoma-specific diagnostic biomarkers. Genome Med [Internet]. 2018 [cited 2021 Sep 30];10:42. Available from:

https://www.ncbi.nlm.nih.gov/pmc/articles/PMC5977535/

11. Dos Santos PW da S, Machado ART, De Grandis RA, Ribeiro DL, Tuttis K, Morselli M, et al. Transcriptome and DNA methylation changes modulated by sulforaphane induce cell cycle arrest, apoptosis, DNA damage, and suppression of proliferation in human liver cancer cells. Food Chem Toxicol. 2020;136:111047.

12. Acunzo M, Romano G, Wernicke D, Croce CM. MicroRNA and cancer-a brief overview. Adv Biol Regul. 2015;57:1-9.

13. Deng S, Calin GA, Croce CM, Coukos G, Zhang L. Mechanisms of microRNA deregulation in human cancer. Cell Cycle. 2008;7:2643-6.

14. Ferreira Martins NN, da Silva Oliveira KC, Braga Bona A, de Arruda Cardoso Smith M, Ishak G, Assumpção PP, et al. The Emerging Role of miRNAs and Their Clinical Implication in Biliary Tract Cancer. Gastroenterol Res Pract. 2016;2016:9797410.

15. Zhang X, Peng Y, Jin Z, Huang W, Cheng Y, Liu Y, et al. Integrated miRNA profiling and bioinformatics analyses reveal potential causative miRNAs in gastric adenocarcinoma. Oncotarget. 2015;6:3287889.

16. Sun Z, Shi K, Yang S, Liu J, Zhou Q, Wang G, et al. Effect of exosomal miRNA on cancer biology and clinical applications. Mol Cancer. 2018;17:147.

17. He J-H, Han Z-P, Zou M-X, Wang L, Lv YB, Zhou JB, et al. Analyzing the LncRNA, miRNA, and mRNA Regulatory Network in Prostate Cancer with Bioinformatics Software. J Comput Biol. 2018;25:14657.

18. Shin VY, Chu K-M. MiRNA as potential biomarkers and therapeutic targets for gastric cancer. World J Gastroenterol. 2014;20:10432-9.

19. Tomczak K, Czerwińska P, Wiznerowicz M. The Cancer Genome Atlas (TCGA): an immeasurable source of knowledge. Contemp Oncol (Pozn). 2015;19:A68-77.

20. Ritchie ME, Phipson B, Wu D, Hu Y, Law CW, Shi W, et al. limma powers differential expression analyses for RNA-sequencing and microarray studies. Nucleic Acids Res. 2015;43:e47.

21. Ito K, Murphy D. Application of ggplot2 to Pharmacometric Graphics. CPT Pharmacometrics Syst Pharmacol. 2013;2:e79.

22. Ru Y, Kechris KJ, Tabakoff B, Hoffman P, Radcliffe RA, Bowler R, et al. The multiMiR R package and database: integration of microRNA-target interactions along with their disease and drug associations. Nucleic Acids Res. 2014;42:e133.

23. Yu G, Wang L-G, Han Y, He Q-Y. clusterProfiler: an R package for comparing biological themes among gene clusters. OMICS. 2012;16:284-7. 
24. Kanehisa M, Goto S. KEGG: kyoto encyclopedia of genes and genomes. Nucleic Acids Res. 2000;28:27-30.

25. The Gene Ontology Consortium. The Gene Ontology Resource: 20 years and still GOing strong. Nucleic Acids Res. 2019;47:D330-8.

26. Szklarczyk D, Gable AL, Lyon D, Junge A, Wyder S, Huerta-Cepas J, et al. STRING v11: protein-protein association networks with increased coverage, supporting functional discovery in genome-wide experimental datasets. Nucleic Acids Res. 2019;47:D607-13.

27. Chen $\mathrm{H}$, Boutros PC. VennDiagram: a package for the generation of highly-customizable Venn and Euler diagrams in R. BMC Bioinformatics. 2011;12:35.

28. Shannon P, Markiel A, Ozier O, Baliga NS, Wang JT, Ramage D, et al. Cytoscape: a software environment for integrated models of biomolecular interaction networks. Genome Res. 2003;13:2498-504.

29. Alimbetov D, Askarova S, Umbayev B, Davis T, Kipling D. Pharmacological Targeting of Cell Cycle, Apoptotic and Cell Adhesion Signaling Pathways Implicated in Chemoresistance of Cancer Cells. Int J Mol Sci. 2018;19:E1690.

30. Hoppe MM, Sundar R, Tan DSP, Jeyasekharan AD. Biomarkers for Homologous Recombination Deficiency in Cancer. J Natl Cancer Inst. 2018;110:704-13.

31. Wang $L$, Mo $C$, Wang $L$, Cheng $M$. Identification of genes and pathways related to breast cancer metastasis in an integrated cohort. Eur J Clin Invest. 2021;51:e13525.

32. Stolfi C, Marafini I, De Simone V, Pallone F, Monteleone G. The Dual Role of Smad7 in the Control of Cancer Growth and Metastasis. International Journal of Molecular Sciences [Internet].

Multidisciplinary Digital Publishing Institute; 2013 [cited 2022 Feb 11];14:23774-90. Available from: https://www.mdpi.com/1422-0067/14/12/23774

33. LiuJingjing, ZhouYang, ShiZhendong, HuYunhui, MengTingting, ZhangXiaobei, et al. microRNA-497 Modulates Breast Cancer Cell Proliferation, Invasion, and Survival by Targeting SMAD7. DNA and Cell Biology [Internet]. Mary Ann Liebert, Inc. 140 Huguenot Street, 3rd Floor New Rochelle, NY 10801 USA; 2016 [cited 2022 Feb 11]; Available from:

https://www.liebertpub.com/doi/abs/10.1089/dna.2016.3282

34. Vishal M, Vimalraj S, Ajeetha R, Gokulnath M, Keerthana R, He Z, et al. MicroRNA-590-5p Stabilizes Runx2 by Targeting Smad7 During Osteoblast Differentiation. Journal of Cellular Physiology [Internet]. 2017 [cited 2022 Feb 11];232:371-80. Available from:

https://onlinelibrary.wiley.com/doi/abs/10.1002/jcp.25434

35. Murugaiyan G, Cunha AP da, Ajay AK, Joller N, Garo LP, Kumaradevan S, et al. MicroRNA-21 promotes Th17 differentiation and mediates experimental autoimmune encephalomyelitis. J Clin Invest [Internet]. American Society for Clinical Investigation; 2015 [cited 2022 Feb 11];125:1069-80. Available from: https://www.jci.org/articles/view/74347

36. $\mathrm{Hu} Y, \mathrm{He} J, \mathrm{He} \mathrm{L}, \mathrm{Xu} \mathrm{B}$, Wang Q. Expression and function of Smad7 in autoimmune and inflammatory diseases. J Mol Med (Berl) [Internet]. 2021 [cited 2022 Feb 11];99:1209-20. Available from: 
https://www.ncbi.nlm.nih.gov/pmc/articles/PMC8367892/

37. Bao Z, Hua L, Ye Y, Wang D, Li C, Xie Q, et al. MEF2C silencing downregulates NF2 and E-cadherin and enhances Erastin-induced ferroptosis in meningioma. Neuro Oncol. 2021;23:2014-27.

38. Di Giorgio E, Wang L, Xiong Y, Christensen LM, Akimova T, Han R, et al. A Biological Circuit Involving Mef2c, Mef2d, and Hdac9 Controls the Immunosuppressive Functions of CD4 + Foxp3 + TRegulatory Cells. Front Immunol. 2021;12:703632.

39. Li Y, Wang Z, Zhao F, Zeng J, Yang X. MicroRNA-190b expression predicts a good prognosis and attenuates the malignant progression of pancreatic cancer by targeting MEF2C and TCF4. Oncol Rep. 2022;47:12.

40. Sereno M, Haskó J, Molnár K, Medina SJ, Reisz Z, Malhó R, et al. Downregulation of circulating miR 802-5p and miR 194-5p and upregulation of brain MEF2C along breast cancer brain metastasization. Mol Oncol. 2020;14:520-38.

41. Yang Z, Jiang X, Li D, Dong Q, Zhao H, Jiang X. TCF21 inhibits proliferation and chemoresistance through the AKT pathway in human gastric cancer. Gene. 2019;682:42-9.

42. Chen B, Zeng C, Ye Y, Wu D, Mu Z, Liu J, et al. Promoter methylation of TCF21 may repress autophagy in the progression of lung cancer. J Cell Commun Signal. 2018;12:423-32.

43. Wang Y, Wu N, Pang B, Tong D, Sun D, Sun H, et al. TRIB1 promotes colorectal cancer cell migration and invasion through activation MMP-2 via FAK/Src and ERK pathways. Oncotarget. 2017;8:4793142.

44. Shahrouzi P, Astobiza I, Cortazar AR, Torrano V, Macchia A, Flores JM, et al. Genomic and Functional Regulation of TRIB1 Contributes to Prostate Cancer Pathogenesis. Cancers (Basel). 2020;12:E2593.

45. Liu Z-Z, Han Z-D, Liang Y-K, Chen J-X, Wan S, Zhuo Y-J, et al. TRIB1 induces macrophages to M2 phenotype by inhibiting IKB-zeta in prostate cancer. Cell Signal. 2019;59:152-62.

\section{Figures}


A

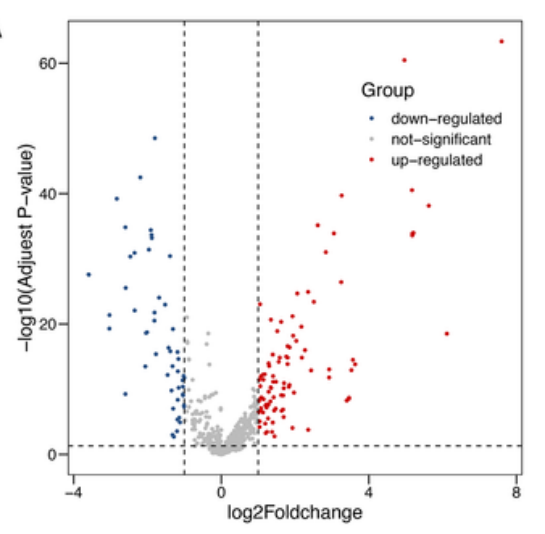

B

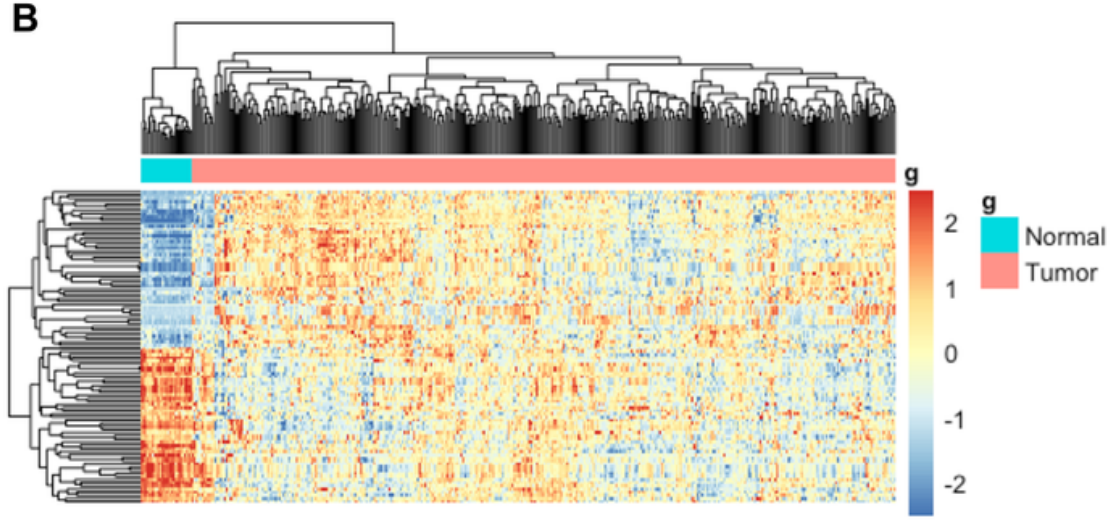

C
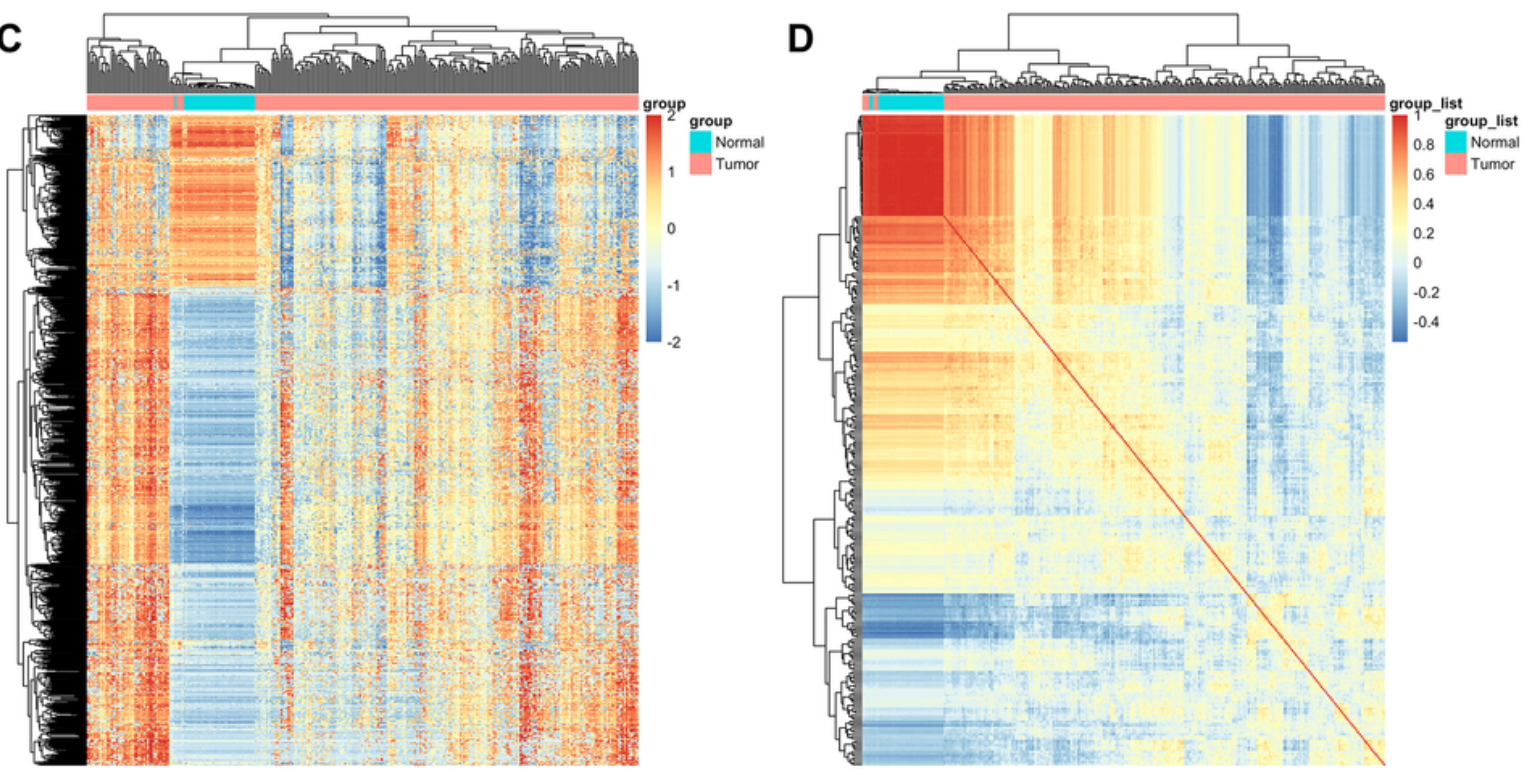

Fig.1

\section{Figure 1}

Identification of DEGs and DMGs between tumor and non-tumor adjacent tissues for datasets from the TCGA-LUSC dataset. (A) Volcano plots of DEMs. X-axis: log2 fold change; Y-axis: -log 10 (P-value) for each gene; vertical-dotted lines: log 2 fold change $\geq 1.0$ or $\leq-1.0$; horizontal-dotted line: the significance cut off (adjusted $P$-value $=0.05$ ). The red dot represents up-regulated genes, and the blue dot represents down-regulated genes. (B) Gene expression heatmap of DEMs for the dataset. (C) Heatmap of the 
differential DNA methylation levels of CpGs. (D) Correlation heatmap of the DNA methylation levels of CpGs within the samples of the TCGA dataset.

A

upDEM-targets

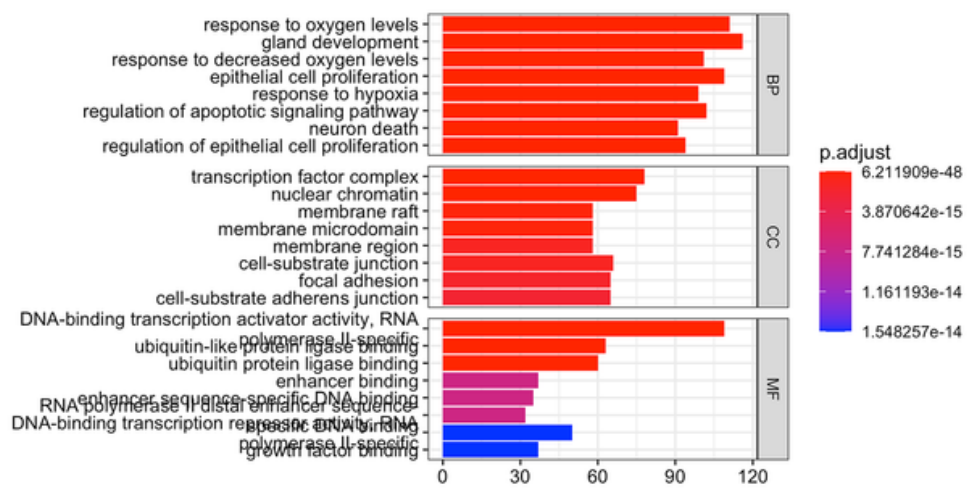

downDEM-targets

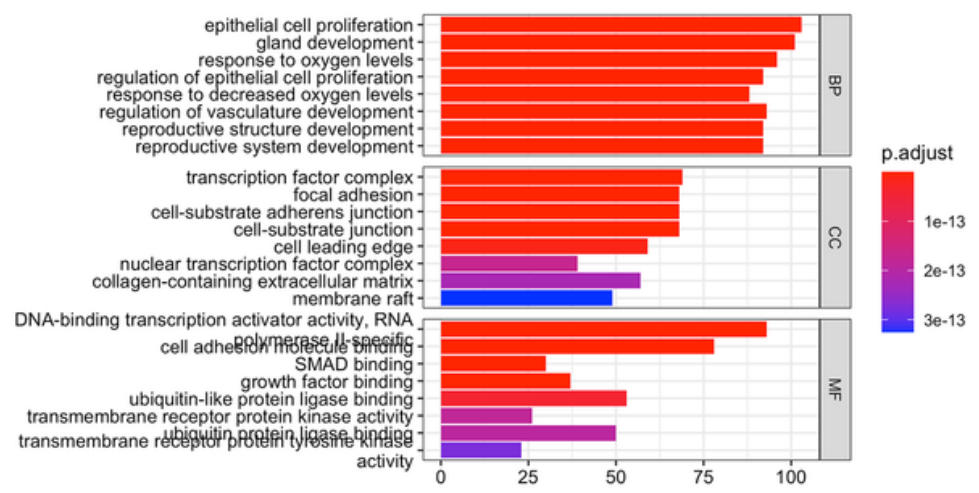

B

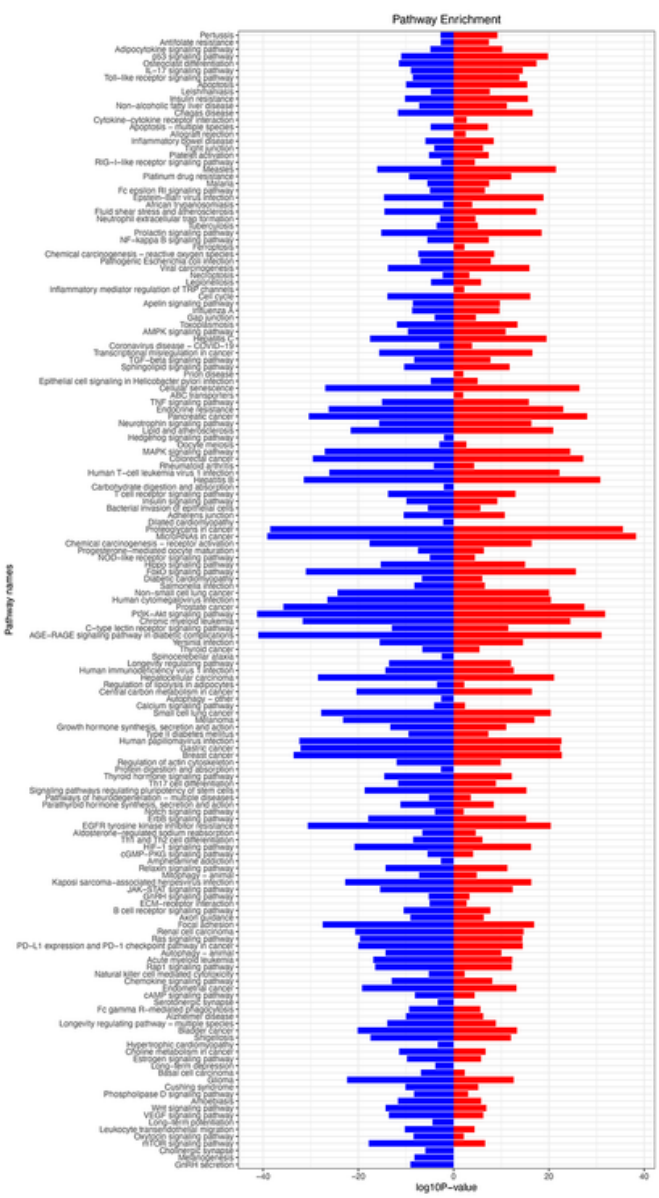

Fig.2

\section{Figure 2}

GO and KEGG annotation analysis of the DEMs targets. (A) the bar plots of the significant GO terms associated with the targets of up-regulated DEMs and down-regulated DEMs, respectively; (B) Pathway enrichment score plots of the targets of up-regulated DEMs and down-regulated DEMs. 


\section{Figure 3}

GO and KEGG annotation analysis of the DMGs located genes. $(A)$ the bar plots of the significant GO terms associated with the located genes of hypermethylated DMGs and hypomethylated DMGs, respectively; (B) Pathway enrichment score plots of the located genes of hypermethylated DMGs and hypomethylated DMGs.

A

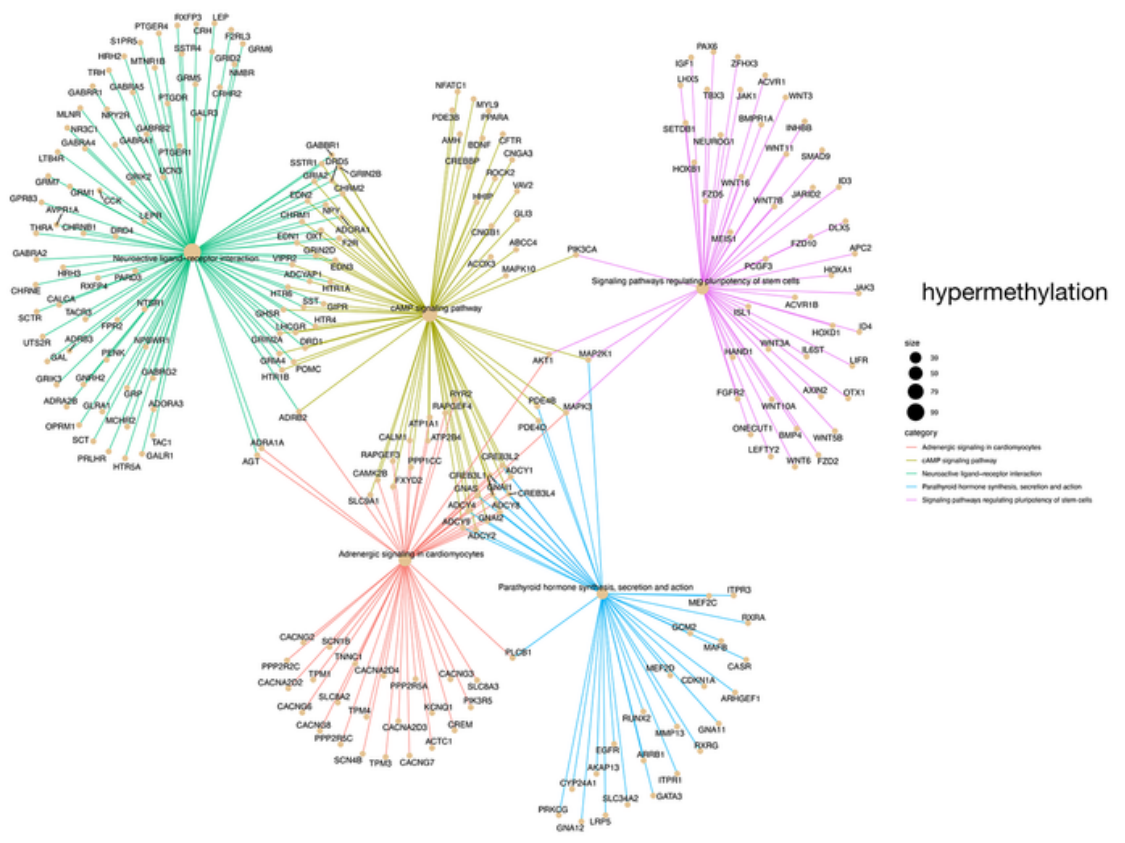

B

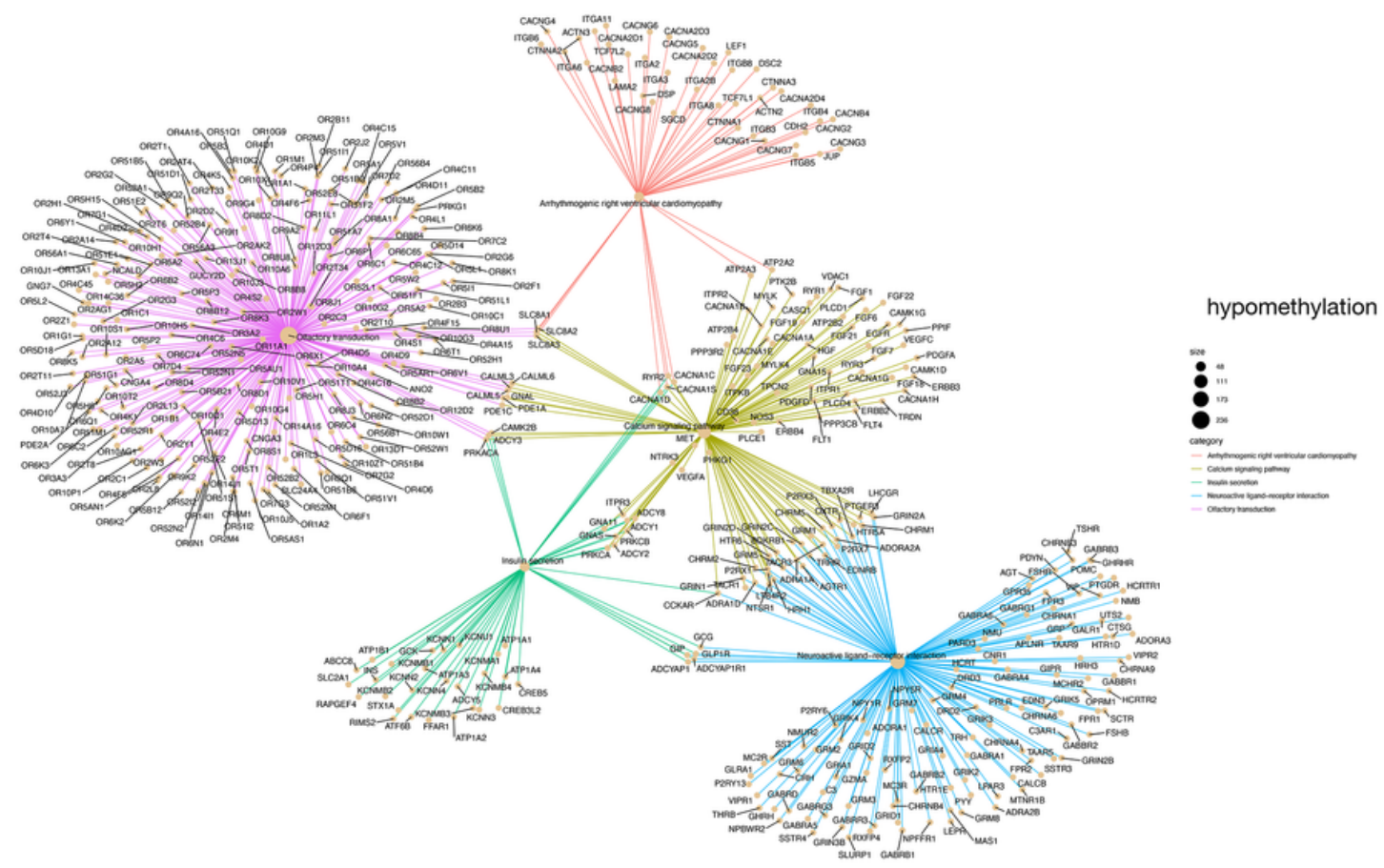

Fig.4 
Figure 4

Cnetplot of pathway enrichment analysis of the located genes of hypermethylated DMGs (A) and hypomethylated DMGs (B).

A

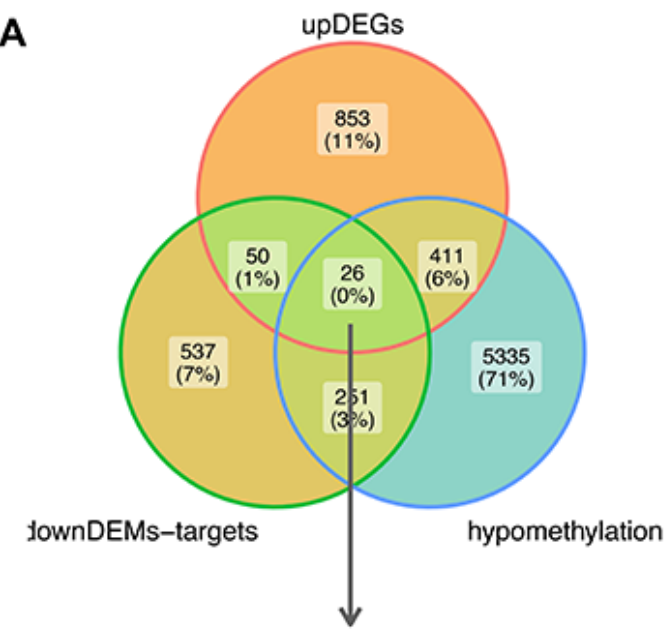

SLC2A1, KIFC1, CDC25A, MCM2, HMGA1, COL7A1, FAM83F, MMP11, ARTN, GAPDH, TERT, HMGA2, SPP1, FSCN1, BCL11A, ADAM12, LGR4, ITGB8, VSNL1, MMP13, JAG1, ADA, CLDN1, SLCO1B1, PITX3, SOST

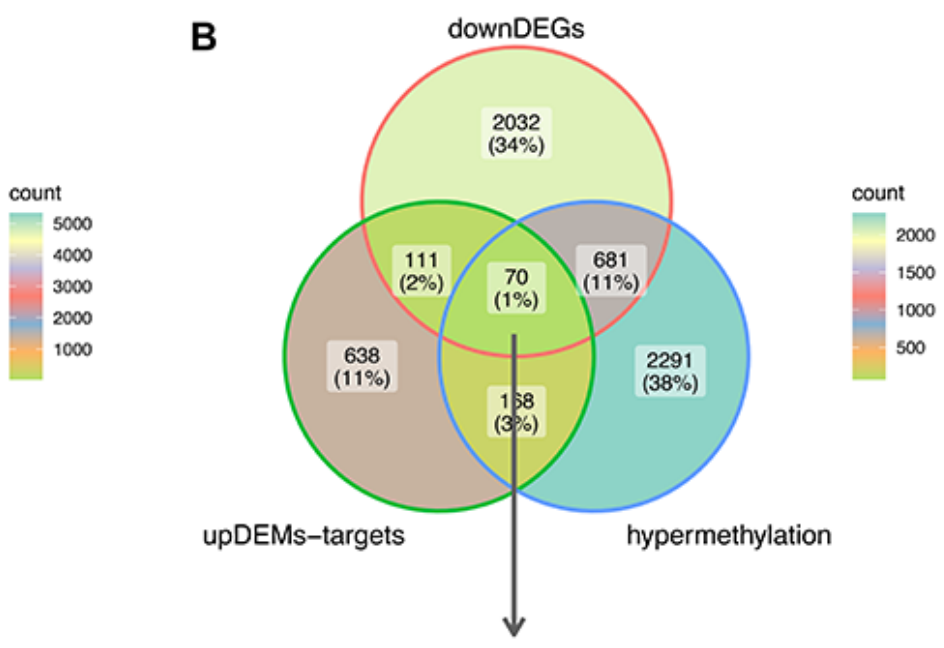

TCF21, SOX17, TMEM88, PRKCE, MAMDC2, LEPR, GPD1L, LRRK2, DOCK4, PTPRM, NROB2, KDR, HECW2, DUSP1, GATA6, SOD3, ALOX5, RBPMS, SPRY4, HNF1B, ZEB2, HOXA5, PRKCZ, KLF9, ITPR1, ZBTB4, RGS5, FOXA2, MEF2C, PRTG, DDAH1, HSPB2, CCND3, SMAD7, FOXP1, LIFR, NRP1, NKD1, TGFBR3, MTHFR, AKAP12, ETS1, MEOX2, DAB2, GAB1, WIF1, BMP2, UBA7, STAT5A, MYLK, TRIB1, ZNF608, TIMP2, NFIB, ZFPM2, HIF3A, PPARGC1A, NTM, CLU, SOX7, TGFB2, MACC1, BDNF, ESR1, ATP8A2, SLC2A3, SOX5, EYA4, TFPI2, DLC1

\section{Figure 5}


located genes; (B) Venn diagrams of the overlapping genes among down-regulated DEGs, up-regulated DEMs targets and hypermethylated DMGs located genes.
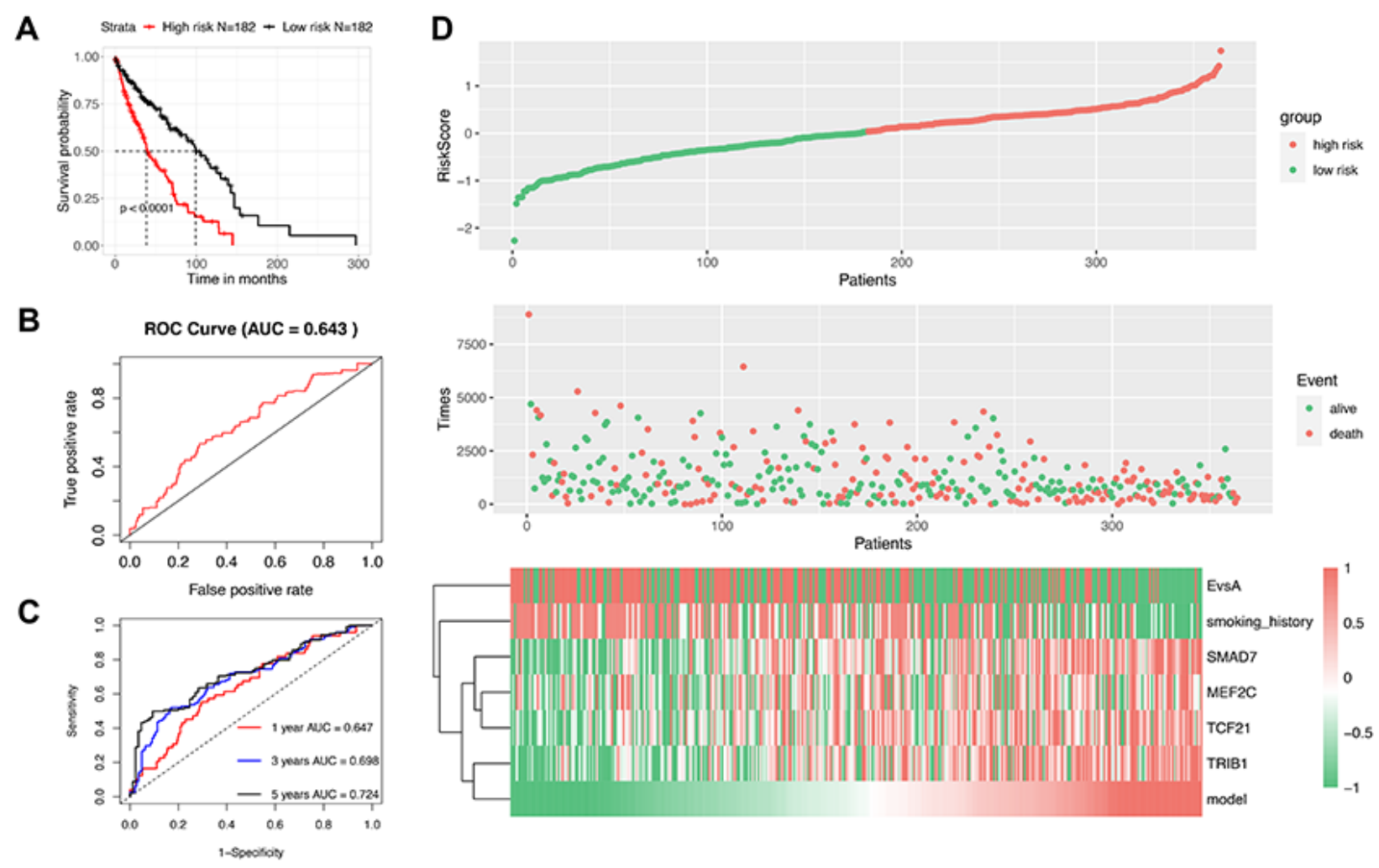

Fig.6

Figure 6

Construction of the prognostic model with DEGs co-regulated by both miRNAs and DNA methylation in TCGA cohort. (A) The Kaplan-Meier survival analysis for overall survival of patients in TCGA cohort; (B) ROC curve analysis of the prognostic value of model; (C) The time-dependent ROC analysis for risk score 
at 1, 3 and 5 years in the TCGA cohort; (D) The heat map of the four genes and the distribution of risk score and the survival status of patients in the TCGA cohort.

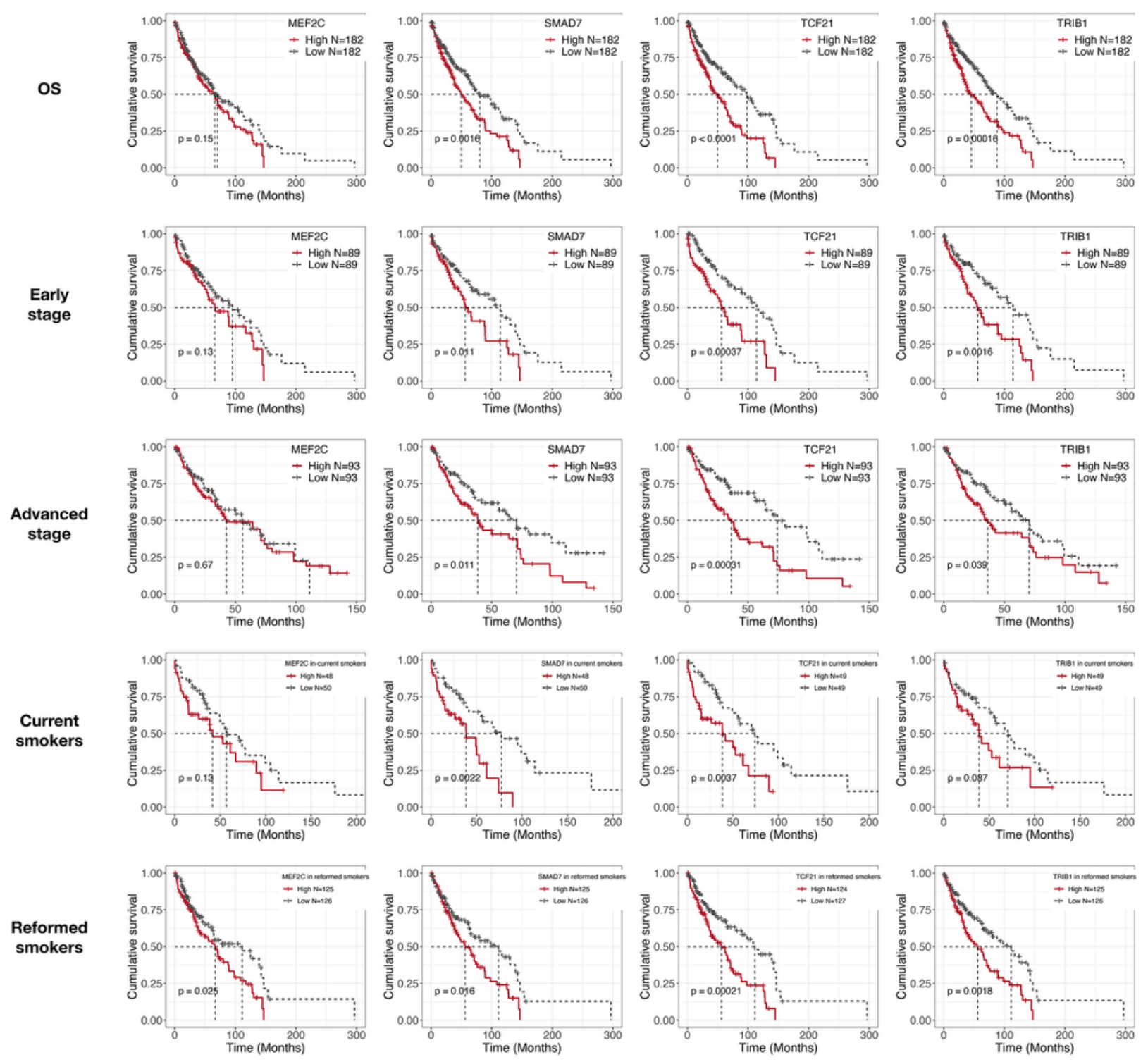

Fig.7

Figure 7

Kaplan-Meier curve and Log-rank test of TCGA samples categorized by the expression of the DEGs in the prognosis model. The patients were stratified into high-level group and low-level group according to median of each gene. 

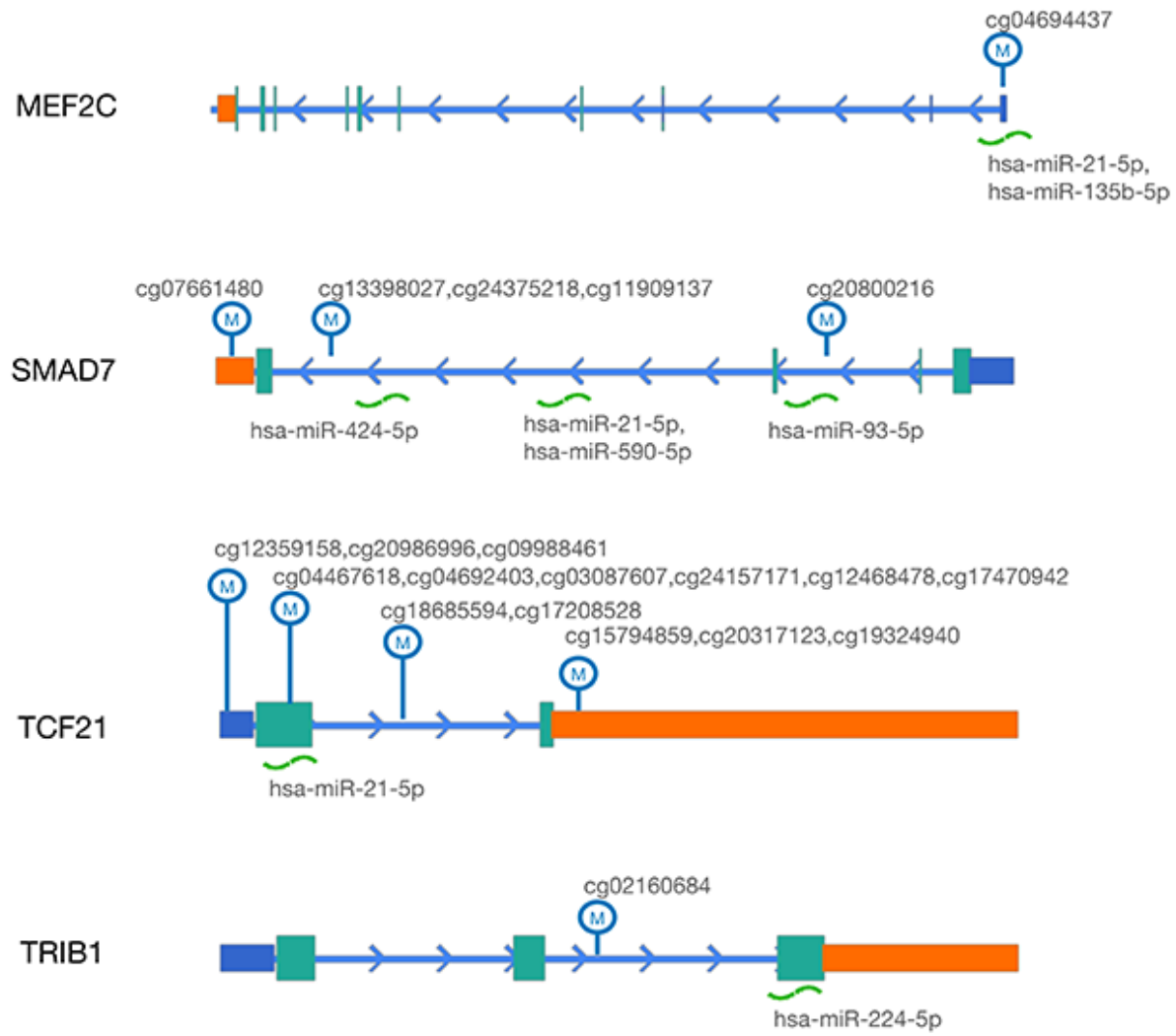

(1) DNA methylation $\frown$ miRNA

Fig.8

Figure 8

Regulation loci of DEGs in the prognosis model regulated by DNA methylation and miRNAs.

\section{Supplementary Files}

This is a list of supplementary files associated with this preprint. Click to download. 
- FigureS1.pdf

- Figures2.pdf

- FigureS3.pdf

- FigureS4.pdf

- FigureS5.pdf

- Tables1.csv

- Tables2.csv

- Tables3.csv

- Tables4.csv

- Tables5.csv

- Tables6.csv

- Tables7.csv

- Tables8.xlsx 